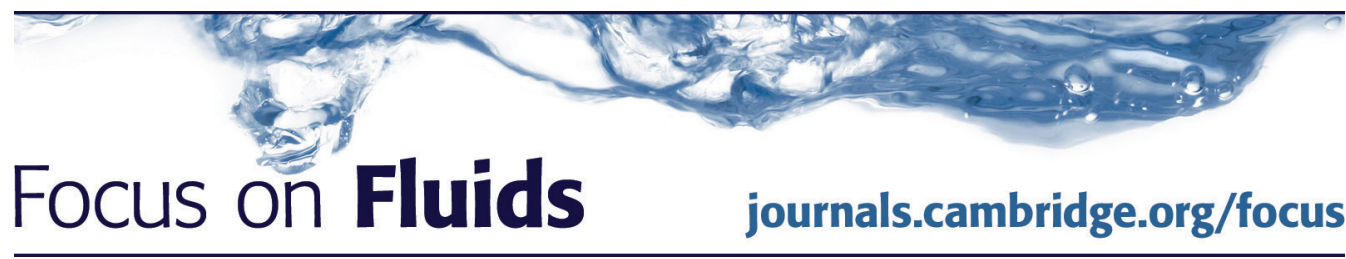

\title{
Anatomy of a diffracting detonation in a circular arc of explosive
}

\author{
John B. Bdzil $\dagger$
}

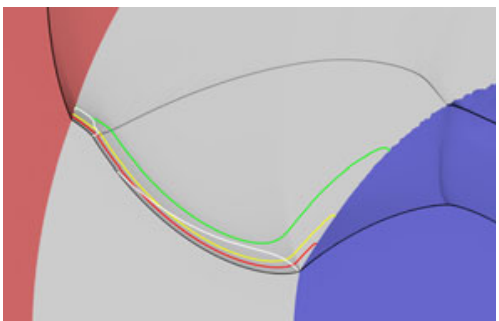

Shock and Detonation Physics Group, Los Alamos

National Laboratory, Los Alamos, NM 87545, USA

Using high-resolution numerical simulation, Short et al. (J. Fluid Mech. vol. 835, 2018, pp. 970-998) study diffraction of a detonation as it traverses a $270^{\circ}$ finitethickness condensed-phase explosive arc. This geometry admits a steady solution in a frame rotating with angular speed $\omega_{0}$, which thereby facilitates a detailed analysis of how the loss of energy from the detonation reaction zone due to the diffraction process slows the propagation of the detonation. There exists a region of subsonic flow, between the detonation shock and the curve of sonic flow (labelled the DDZ), which is responsible for setting $\omega_{0}$. Although the DDZ spans the entire thickness for thin arcs, it is localized to a region near the inside surface as the arc is thickened. Thus the explosive energy release near this inside surface plays a disproportionate role in the diffraction process.

Key words: detonation waves, high-speed flow

\section{Introduction}

We have all watched waves come onto a beach, encounter an object, diffract around that object and weaken. Something similar happens when a high-speed planar square-topped shockwave, running parallel to a rigid wall and in a non-reactive compressible material, encounters a convex corner; the shock diffracts around the corner in a weakened form. Such a two-dimensional flow is scale-free and thus expressible in terms of the two similarity variables, $\xi=x / t, \eta=y / t$. Then, it becomes possible to locate the zone of influence of the corner and define where the flow is subsonic and supersonic, and the separating sonic locus, as one would do for a steady-state two-dimensional high-speed flow. This flow is sketched in figure $1(a)$. The dashed curve denotes the locus of sonic flow in the corner-attached frame and separates the disturbed subsonic flow on the right from the undisturbed flow on the

$\dagger$ Email address for correspondence: jbbdzil@gmail.com 

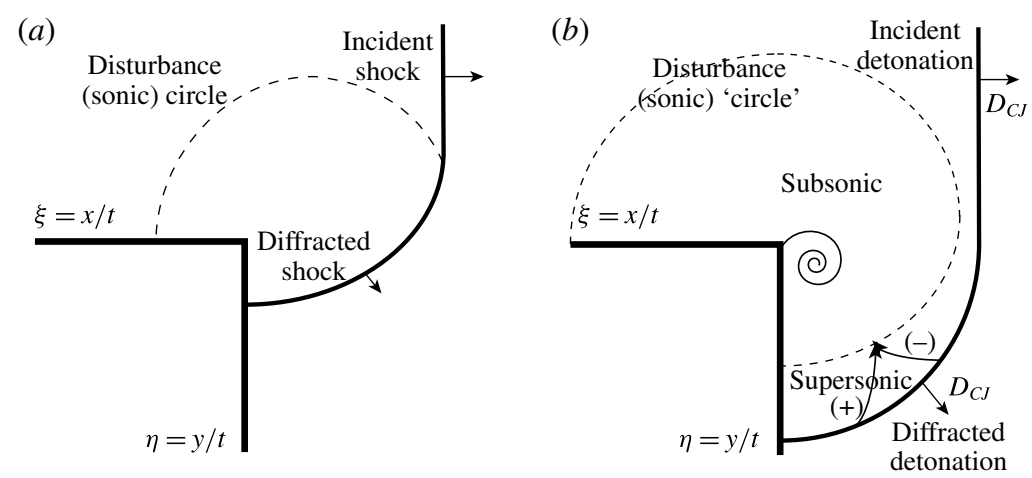

FIGURE 1. A sketch of the main features for inert shock diffraction (a) compared with the solution of detonation diffraction for an idealized CJ explosive $(b)$.

left. Energy flows from the upper undisturbed region into the disturbed (diffracted) region and thus supports the diffracted shock (although at reduced pressures).

Given the same scenario as above, but replacing the inert compressible material with a detonating condensed-phase explosive, we find a somewhat different flow. In detonation, the high-speed shock triggers rapid energy-releasing reactions in the explosive which in turn support the shock. When this reaction zone is very short compared with other problem scales, we can approximate the detonation with the scale-free instantaneous Chapman-Jouguet (CJ) detonation, which yields the diffraction scenario pictured in figure $1(b)$. The detonation propagation speed, $D_{C J}$ (the shock speed in this problem), is the same in both the undisturbed and the diffracted regions, as the instantaneous reaction provides all of the energy needed to fully support the diffracting detonation shock. The locus of sonic flow is again the nearly circular region surrounding the corner. However, now the circular diffracting $D_{C J}$-speed detonation shock is separated from the region of subsonic flow by a supersonic buffer zone, where only information travelling away from the shock, boundary and undisturbed flow defines the solution.

The picture given for the CJ detonation is an idealized description. When the finitethickness reaction zones of real explosives are considered, the description needs to be modified to account for the lateral loss of energy from the reaction zone. This flow is not scale-free, and the diffracting detonation shock speed is reduced to sub- $D_{C J}$ values, resulting in reduced pressures and temperatures in the diffracting reaction zone. In turn, this leads to an increased length of the reaction zone the closer one moves to the corner and the turned wall, where the energy loss due to diffraction is the greatest (see Kapila et al. 2007).

\section{Overview}

This class of sudden convex-corner wall-expanding flows subjects a finite-length reaction zone to diffraction of widely varying strengths, and can include such features as vortices, slip lines, shocks embedded in the interior flow, etc. (see figure $1 b$ and Kapila et al. 2007), which leads to a fully time-dependent two-dimensional flow. Such a scale-dependent flow is not suitable for detailed analysis, since the flow is neither self-similar nor steady. The finite-thickness circular arc explosive geometry studied by Short et al. (2018) represents a controlled-strength diffraction geometry that allows 
(a)

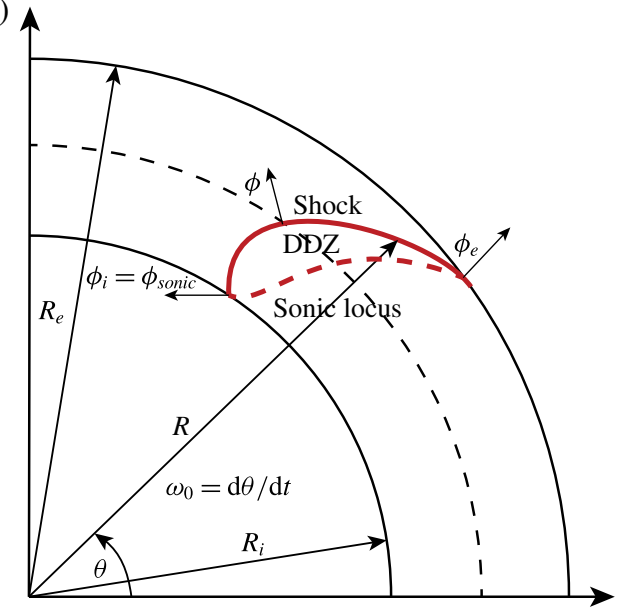

(b)

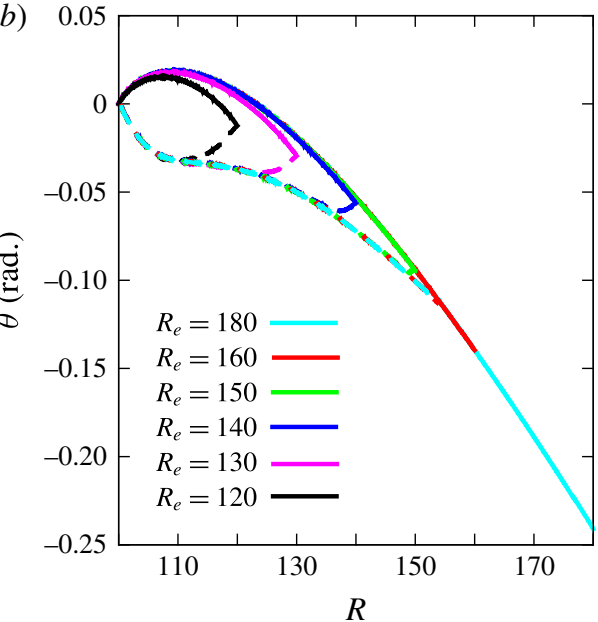

FIGURE 2. A sketch of the explosive-arc geometry used by Short et al. (2018) (a) and results showing the DDZ as a function of the arc dimensions and confinement $(b)$.

the simplification of a steady-state solution in a rotating frame (a frame fixed to the centre of curvature of the cylindrical surfaces of the arc, as displayed in figure $2 a$ ). Experiments by Nakayama et al. (2012) show steady propagation in this geometry. Since the rigid-wall boundary assumption, suitable for gas flows, is inappropriate for condensed-phase high explosives, Short et al. (2018) consider the case of deformable explosive boundary confinement. Now additional reaction zone energy is lost as the confining walls are pushed outward by the detonating high explosive.

What Short et al. (2018) observe in their numerical simulations is the flow becoming steady. Then, it becomes possible to define the subsonic and supersonic regions of the flow. Now, a subsonic region sits behind the detonation shock, and the flow only turns supersonic (as in the instantaneous-reaction CJ detonation case) towards the end of the reaction zone. This subsonic zone defines the rotation speed of the diffracting detonation and is what the authors define as the detonation-driving zone (DDZ). Importantly, although the DDZ extends to the inner surface of the arc, it does not necessarily extend to the outer surface of the arc even though the reaction zone deposits energy there. The factors that influence the extent of the DDZ are the inner and outer radii, $R_{i}$ and $R_{e}$, of the explosive arc and the nature of the materials confining the explosive on the inside and outside cylindrical surfaces of the arc. For this steady flow, the sonic parameter along the shock can be written as

$$
c^{2}-\tilde{u}_{\theta}^{2}-\tilde{u}_{r}^{2}=\left(\left(\frac{\cos \phi}{\cos \phi_{\text {sonic }}}\right)^{2}-1\right)\left(\omega_{0} R\right)^{2},
$$

where the normal detonation speed is given as

$$
D_{n}=\left(\omega_{0} R\right) \cos \phi
$$

where $D_{n}$ is an order-one quantity. Here, $c$ is the sound speed and $\tilde{u}_{\theta}$ and $\tilde{u}_{r}$ are the two components of the particle velocity in the $\theta$ - and $R$-dependent rotating frame; $\omega_{0}$ is the constant angular velocity of the frame, while $\phi$ is the angle between the 
normal to the detonation shock and the normal to $R$ at the shock (see figure $2 a$ ). What (2.1)-(2.2) show is that as $R$ increases, $\cos \phi \rightarrow 0\left(\phi \rightarrow 90^{\circ}\right)$, and the flow becomes supersonic at the outer surface for sufficiently large and finite $R_{e}$. Until this critical $R_{e}$ is reached, the DDZ extends to $R_{e}$, with the details of the DDZ near the outer surface depending on the outer confinement layer properties. Organic solids provide weak confinement and a sonic flow along the outer edge in the rotating frame, while metals provide strong confinement with a subsonic, $D_{n}>D_{C J}$ Mach reflection possible there. Short et al. (2018) show that after the flow at the outer surface has become supersonic, the solution there is independent of the properties of the outer confinement layer. Then, information about the confinement can only flow backwards and away from the front, and thus does not influence the detonation shock. As $R_{e}$ increases beyond the critical transition value, the flow near the outer surface becomes more supersonic and the DDZ recedes from the outer surface irrespective of the nature of the outer confinement layer. A comparison of the DDZ (i.e. the shocks and sonic loci) as $R_{e}$ changes is displayed in Short et al. (2018) figure 17, and is reproduced here as figure 2(b). Thus, as $R_{e}$ increases, the speed of rotation and the shape of the detonation shock are set by only the portion of the reaction zone near $R_{i}$, with the detonation near $R_{e}$ being dragged along by the subsonic flow region (DDZ) that sits near $R_{i}$. Significantly, it is the inner layer of explosive that plays a dominant role in setting the properties of a steadily diffracting detonation in an arc of explosive, as compared with the outer layer playing the dominant role for inert-material shock diffraction.

\section{Future}

One sees that for a fixed $R_{i}$, the angular speed of the diffraction, $\omega_{0}$, depends on the geometry of the DDZ, with $\omega_{0}$ not changing once $R_{e}$ exceeds a critical value, and the DDZ pulls away from the outer surface of the arc. Most importantly, $\omega_{0}$ depends on the details of the flow nearer the inside surface of the arc (see also Short et al. 2016). Only a single value of $R_{i}$ and weak confinement were considered on the inner surface. One can only speculate that as $R_{i}$ and the inner surface confinement change, the DDZ will remain attached to the inner arc surface. This leaves open how $\omega_{0}$ and the DDZ change as $R_{i}$ is decreased, which increases the flow divergence, to the point where detonation near the edge is extinguished. Then, at some critical $R_{i}$, the reaction zone would decouple from the shock near the inner edge, and the energy release in the interior of the arc would play the dominant role in setting $\omega_{0}$. Both the poorly understood problems of detonation extinction and detonation Mach reflection could greatly benefit from being studied as steady-state flows that the arc geometry supports.

\section{References}

Kapila, A. K., Schwendaman, D. W., Bdzil, J. B. \& Henshaw, W. D. 2007 A study of detonation diffraction in the ignition-and-growth model. Combust. Theor. Model. 11 (5), $781-822$.

Nakayama, H., Moriya, T., Kasahara, J., Matsuo, A., Sasamoto, Y. \& Funaki, I. 2012 Stable detonation wave propagation in rectangular-cross-section curved channels. Combust. Flame 159, 859-869.

Short, M., Quirk, J. J., Chiquete, C. \& Meyer, C. D. 2018 Detonation propagation in a circular arc: reactive burn modelling. J. Fluid Mech. 835, 970-998.

Short, M., Quirk, J. J., MeYer, C. D. \& Chiquete, C. 2016 Steady detonation propagation in a circular arc: a detonation shock dynamics model. J. Fluid Mech. 807, 87-134. 